\title{
Geschichte der Pädagogik im Curriculum der Lehrerausbildung für Grund- und Bürgerschulen (tschechisch-deutscher Vergleich: 1918-1939) - eine wissenschaftliche Disziplin im Dienste des Volkes, des Fortschrittes und der Sozialreform
}

\author{
TOMÁš KasPer
}

Abstract: The article focuses on the analysis of course books concerning the history of pedagogy used in the interwar period at the Czech and German Teachers' Institutions. Based on the analysis, there is pointed out the role of the subject concerning the history of pedagogy in the given period in the framework of the teachers' training.

The analysis is set into the wider context of the development concerning pedagogical thinking and its reflection in the Czech and German pedagogical debate at the turn of the 19th and 20th centuries in the Czech lands of the monarchy and the first half of the 20th century in the interwar Czechoslovakia.

The analysis not only points to a different grasp of the historical reflection of pedagogical thinking in both national and cultural camps, but also points to common features in the development of the discipline.

These are mainly shown in its functional character, when the history of pedagogy was a discipline supporting the national emancipation of both nations, and they were part of a wider debate concerning reform of the society.

Keywords: history of education, teachers' institutes, course books, teachers' training, Czechoslovakia, Czech Lands and the Habsburg Monarchy.

\section{EINLEITUNG}

Die Geschichte der Pädagogik als Lehrfach spielte bereits ab dem Jahr $1869^{1}$ im Bildungskonzept für Lehrkräfte in Grund- und Bürgerschulen, im Rahmen des vierjährigen Curriculums Pädagogischer Institute, eine bedeutende Rolle.

\footnotetext{
${ }^{1}$ Im Jahre 1869 trat das sogenannte Hasners Schulgesetz in Kraft, in welchem u. a. die Einrichtung vierjähriger LehrerInnenbildungsanstalten zur Vorbereitung von Lehrkräften für Grund- und Bürgerschulen verankert wurde.
} 
Nicht anders war die Lage in der gesamten Zwischenkriegszeit, welche die „Tradition der Geschichte der Pädagogik" als wichtigen Bestandteil der Lehrer-Ausbildung kontinuierlich übernahm. Ebenso hohen Stellenwert hatte die Disziplin im Rahmen der pädagogischen Ausbildung von Mittelschullehrern im Vortrags-Portfolio der Karl-Ferdinands-Universität (und nach deren Teilung in die tschechische und die deutsche Universität 1882 - in beiden Universitätsteilen), als auch nach 1918 an der tschechischen Karls-Universität in Prag und der Deutschen Karls-Universität in Prag. Diese Feststellung kann bei Pädagogischen Instituten durch ihren Lehrplan belegt werden, welcher - nach 1874 konsolidiert und nach 1918 reformiert - das Unterrichtsfach Geschichte der Pädagogik als wesentlichen Bestandteil der Bildungsinhalte wahrte. Die Position der Geschichte der Pädagogik in der Ausbildung von Mittelschulprofessoren kann durch Vortragsverzeichnisse beider Prager-Universitäten nachgewiesen werden. Wenn wir uns jedoch nicht mit einem rein quantitativen Blick auf die Stellung der Geschichte der Pädagogik in Lehrplänen, bzw. in der Struktur eines Universitätsstudiums oder -Vorträgen begnügen wollen und uns die Frage stellen, welche Ziele mit Unterricht und Vorträgen über die Geschichte der Pädagogik verfolgt wurden, wie Lehrbücher zur Geschichte der Pädagogik für pädagogische Institute konzipiert wurden, oder welche Ausrichtung fachliche pädagogische Studien hatten, die als Basis für Vorträge dienten, dann wird es erforderlich, ein Korpus aus grundlegenden LehrbuchSchriften und Fachstudien zu lokalisieren und dessen Konturen zu definieren. Folglich werden solche Werke analytisch und mithilfe nachstehender Prüfungsfragen untersucht. ${ }^{2}$ Welche Ziele verfolgten Lehrbücher zur Geschichte der Pädagogik für pädagogische Institute? Wie wurde ein „Kanon der Klassiker der Pädagogik“ festgelegt, welche für den Saal der Geschichte der Pädagogik ausgewählt wurden? Wie wurde eine solche Auswahl von deren Autoren begründet und legimitiert? Findet man Unterschiede in der Überlieferung und im Konzept des Unterrichtsfaches und in der Disziplin Geschichte der Pädagogik in Debatten tschechischer und deutscher pädagogischer Experten? Wenn ja, worin lagen sie und worin stimmten sie überein?

Noch umfassender ausgerichtet ist unsere Frage, ob tschechische und deutsche Lehrbücher für zukünftige Lehrer an Volks- und Bürgerschulen in den Böhmischen Ländern der Monarchie und in der Tschechoslowakischen Republik ein Beispiel der derzeit verbreiteten

\footnotetext{
${ }^{2}$ Vorliegende Abhandlung beschränkt sich auf die Komparation der Definition und der Auffassung von der Geschichte der Pädagogik im Rahmen des Studiums in Pädagogischen Instituten. Eine Analyse dieser Disziplin im Rahmen eines Universitätsstudiums als Vorbereitung von mittelschulischen Lehrer ist nicht miteinbezogen. Historisch-pädagogische Schriftstücke, die als Grundlage für Vorträge von Pädagogik-Professoren an tschechischen Universitäten in Prag und Brünn, als auch auf der Deutschen Universität in Prag dienten, werden deshalb nicht untersucht.
} 
Anschauung der Geschichte des pädagogischen Denkens darstellen, oder ob die Entwicklung des Bildungswesens, dessen didaktischer Methoden, Formen des Unterrichts etc. im historischen Kontext breiterer sozio-politischer, kultureller und wissenschaftlicher Diskussionen gesehen wurden. Des Weiteren wollen wir wissen, ob Lehrbuch-Autoren auf die von ihnen ausgewählten und in deren Büchern miteinbezogenen pädagogischen Richtungen einen offenen Blick geboten haben. Oder, ob sie diese für „auf selbstverständliche und natürliche“ Weise erscheinende pädagogische Strömungen hielten? Über die Einführung solcher Autoren in den Saal der Klassiker der Pädagogik wird wahrscheinlich kaum jemand nachgedacht haben und kaum jemand wird sie auf irgendeine Weise auch gerechtfertigt haben wollen. Deshalb stellen wir uns die Frage, ob wir auch im mitteleuropäischen Raum vor allem ein Aufschreiben der "Geschichte der Geschichte der Pädagogik" erleben, oder ob der Kanon pädagogischer Schulen einer tieferen Reflexion und Untersuchung unterzogen wurde? (Tenorth, 1996; Depaepe, 2001) Wir wagen uns zu hinterfragen, inwieweit sich LehrbuchAutoren bemühten, verschiedene Lesearten (different reading), sprich auch verschiedene Schreibarten (different writing) ausgesuchter pädagogischer Probleme anzubieten. Oder ob wir, was anzunehmen wäre, eine undifferenzierte und einheitliche Sichtweise dessen erleben, was die Geschichte der Pädagogik darstellt und was sie sagt?
Wir fragen uns, ob die Geschichte der Pädagogik den wissenschaftlichen Charakter der Pädagogik zu bestätigen hatte, oder ob ihre Aufgabe als Disziplin darin lag, die herrschende Sozialordnung bei den zukünftigen Lehrkräften zu legitimieren? Das letztere würde bedeuten, dass die geschichtlich-pädagogische Forschung über die Grenzen einer Wissenschaft mit der Aufgabe hinaus gegangen gewesen wäre, einer sozialen Ordnung Legitimität zu verschaffen. (Tröhler, 2014, 2017) Es sollte in diesem Zusammenhang nicht vergessen werden, dass die deutsche Überlieferung der Geschichte der Pädagogik oder die eigene Mentalkraft sich aus der Tradition des letzten Drittels des 19. Jahrhunderts entwickelte und daraus schöpfte. Sprich aus jener Zeit, in der sich pädagogische Wissenschaft und Lehrerbildung in einer engen Verbindung mit Forderungen gesellschaftlicher Ordnung der Habsburgermonarchie des Endes des 19. Jahrhunderts befand. Diese Tradition wurde nach der Gründung der Tschechoslowakischen Republik (ČSR) durch Werte einer republikanischen Ordnung stark erschüttert. Ihre Werte hätten eine Unterstützung insbesondere in der tschechischen pädagogischen Elite durch eine Neukonzeption und ein neues Ausbildungssystem der Lehrer finden sollen, in welchem die Geschichte der Pädagogik zu einem differenzierteren Anschauen von Erziehungs- und Bildungsprozessen angesichts deren gesellschaftlicher Bedingtheit zu führen hatte. 
Es stellt sich die Frage, ob die in den Pädagogischen Instituten Böhmischer Länder und vor allem in der ČSR verwendeten tschechischen und deutschen Lehrbücher, aus einer "nationalen“ Sicht doch bestimmte Unterschiede untereinander aufweisen. Es ist anzunehmen, dass die Geschichte der Pädagogik, welche als Anker des wissenschaftlichen Charakters der sich damals entwickelnden Pädagogik diente, die wiederum selbst ein Mittel zur nationalen und sozio-politischen Emanzipation beider Völker Böhmischer Länder zu sein hatte, ein Korpus an Ideen und Persönlichkeiten in Zusammenhang mit „national-emanzipatorischer Aufgabe" und mit der präferierten Sozialordnung definiert hat (Kasper \& Kasperová, 2013).

\section{GeschichtLicher RAHMEN} „TSCHECHISCHER UND DEUTSCHER “ PäDAGOGIK IN BÖHMISCHEN

\section{LÄNDERN DER MONARCHIE UND IN DER ČSR}

Es ist daher angezeigt sich die Frage zu stellen, ob in der Auffassung der Geschichte der Pädagogik in den auf Tschechisch und Deutsch geschriebenen Lehrbüchern für Pädagogische Institute, Unterschiede $\mathrm{zu}$ finden waren. Lehrbücher sollen einerseits eine didaktische Transformation des Lehrstoffes gewährleisten, anderseits und vor allem aber stellen sie das Ergebnis eines bestimmten „wissenschaftlichen Konsensus“ und einer Sozialordnung dar, welches sich in der Festlegung der zu unterrichtenden
Materie und der Bildungsziele äußert. Deshalb ist davon auszugehen, dass tschechische und deutsche Lehrbücher zur Geschichte der Pädagogik für Pädagogische Institute an der Wende zum 20. Jahrhundert und für die Zwischenkriegszeit teilweise ähnlich und teilweise auch unterschiedlich konzipiert wurden. Dies lässt sich damit erklären, wie diese Disziplin als Bestandteil der ganz jungen pädagogischen Wissenschaft in der tschechischen und der deutschen pädagogischen Diskussion thematisiert wurde, bzw. wie sich die Pädagogik als Wissenschaft in beiden fachlichen pädagogischen Volkslagern aufgebaut hat. Prag wurde zum wissenschaftlichen Mittelpunkt der pädagogischen Debatte vor Gründung der Tschechoslowakei. Und genau dort, auf der nach nationalem Prinzip in eine tschechische- und eine deutsche Universität aufgeteilten KarlFerdinands-Universität in Prag, ist es klar, welch unterschiedliche Sichtweisen es zwischen der tschechischen und der deutschen pädagogischen Elite im Bezug auf Pädagogik als Lehrfach und vor allem auf wissenschaftliche Forschungsmethode gab (Kasper \& Kasperová, 2015, 2017). So begab sich die tschechische pädagogische Wissenschaft nach der Epoche des Herbartianismus (seit den 70er Jahren des 19. Jahrhunderts) auf die Suche nach neuen Vorbilder für die Entwicklung der pädagogischen Debatte und wurde vor allem im englischen Positivismus von Herbert Spencer fündig, aber auch in Einflüssen des Evolutionismus von Charles Darwin auf Fragen über die Er- 
ziehung des Menschen (Werk der Prager Pädagogik-Professoren G. A. Lindner, ${ }^{3}$ J. Durdík ${ }^{4}$ und P. Durdík, ${ }^{5}$ oder über die Bedeutung von maßgeblichen positivistisch orientierten Lehrern und vor allem von J. Úlehla $\left.{ }^{6}\right)$. Die deutsche Pädagogik in Prag entwickelt demgegenüber (in erster Reihe im Werke von O. Willmann ${ }^{7}$ ) eine normativ basierte Auffassung pädagogischer Wissenschaft, beeinflusst u. a. durch Werte der katholischen Kirche.

Während tschechische Professoren unter dem Einfluss des Positivismus eine
Entkirchlichung der Schule und Entflechtung von Kirche und Staat auf dem Gebiete der Bildung und Erziehung fördern, zeigt sich die deutsche pädagogische Repräsentation in Bezug auf die Verknüpfung zwischen Erziehung und Religion vielmehr kompromissbereit. Man kann also feststellen, dass Böhmische Länder an der Wende zum 20. Jahrhundert zu einem höchst interessanten intellektuellen Gebiet wurden, in dem zwei unterschiedliche Traditionen von Wahrnehmung der Pädagogik als Wissenschaft

${ }^{3}$ G. A. Lindner (1828-1887) wurde 1882 zum ersten Pädagogik-Professor, der auf der neu gegründeten tschechischen Karl-Ferdinands-Universität in Prag Vorträge auf Tschechisch hielt. Zu diesem Zeitpunkt war er bereits ein weit bekannter Lehrbuchautor zur Allgemeinen Pädagogik. Seine Lehrbücher wurden sowohl in deutsch- und tschechischsprachigen Pädagogischen Instituten der Habsburgermonarchie, als auch in Deutschland verbreitet (Allgemeine Erziehungslehre, 1877, und Allgemeine Unterrichtslehre, 1877). Vor allem das Spätwerk G. A. Lindners dient als Beispiel seines Abhebens von J. F. Herbarts Lehre und als Muster einer durchdachten pädagogischen Konzeption, geführt im Geiste des Positivismus und insbesondere nach dem Vorbild von H. Spencers Ideen. In Fragen natürlicher Entwicklung beruft sich G. A. Lindner unter anderem auf Ch. Darwin. In Frage kultureller und moralischer Entwicklung beruft er sich auf Auguste Comte.

${ }^{4}$ Josef Durdík (1837-1902) war Ästhetik- und Pädagogikvortragender und Nachfolger von G. A. Lindner auf der Karl-Ferdinands-Universität in Prag im Zeitraum 1888-1899. Neben dem Einfluss von Herbarts Lehre weist Durdíks Werk vor allem Interesse für die Lehre von Ch. Darwin auf, den Durdík persönlich 1875 während seiner Studienreise durch England besuchte und dessen Werk er übersetzte. Evolutionismus und Entwicklungsgesetz durchzogen Durdíks Denkweise deutlich und prägten u. a. seine Konzeption der Erziehungs- und Sittenlehre sowie der Ästhetik.

${ }^{5}$ Petr Durdík (1845-1909) war Bruder von J. Durdík und Vortragender mittelschulischer Pädagogik an der Prager-Universität bis Ende des 19. Jahrhunderts. Seine Auffassung von Pädagogik und vor allem der Sittlichkeit war durch einen positivistischen und evolutionistischen Ansatz stark geprägt.

${ }^{6}$ Josef Úlehla (1852-1933) war ein bedeutender Lehrer der Bürgerschule und ein hoch aktiver Funktionär in Lehrervereinigungen am Ende des 19. Jahrhunderts und im ersten Drittel des 20. Jahrhunderts. J. Úlehla brachte einige Konzepte von Schulreform hervor, die im Sinne der Tatschule und differenzierter Einheitsschule geführt wurden. Úlehla stützte seine Reformkonzepte auf seine eigenen theoretisch-pädagogischen Schriften, aufgebaut auf Ch. Darwins und H. Spencers Lehre. J. Úlehla übersetzte Schriftstücke von H. Spencer für die tschechische Lehrerschaft und wurde zu einem aktiven Kritiker „deutscher Schule“ und „deutscher Pädagogik“. Für die Weiterentwicklung tschechischer Pädagogik sieht er den einzigen Weg in der Befreiung vom philosophischen und geisteswissenschaftlichen Einfluss deutscher Pädagogik und in einer Annäherung an die englische positivistische Pädagogik. (H. Spencer, J. S. Mill, J. Locke). In diesem Sinne wirkte Úlehla auf die tschechische Lehrerschaft durch seine Aktivität in Vereinen und durch die Ausgabe von Schriften und pädagogischen Zeitschriften.

${ }^{7}$ Otto Willmann (1839-1920) kam 1872 als Professor für Theologie und Pädagogik aus Wien. Als ordentlicher Professor blieb er bis 1903 tätig. Nach seiner Pensionierung wirkte er als Bischof in Leitmeritz. 
auf einem „kleinen Raum“ aufeinander trafen. Und nicht nur das. Es zeichnen sich auch unterschiedliche Perspektiven in Verbindung mit der Aufgabe der Wissenschaft im Allgemeinen ab, dies in einem größeren gesellschaftlichen Zusammenhang, was auch einen erheblichen Einfluss auf die Erziehung der nachwachsenden Lehrergeneration hatte. Folglich können Lehrbücher zur Geschichte der Pädagogik gleichzeitig als ein Medium betrachtet werden, welches diese wissenschaftliche Unterschiedlichkeit gewissermaßen spiegelt und belegt.

Auch nach der Gründung der Tschechoslowakischen Republik strebte sowohl die tschechische als auch die deutsche Pädagogik eine Legitimierung gesellschaftlich sowie politisch anderweitiger Ziele an. Vereinfacht lässt sich feststellen, dass tschechische Pädagogik auf der Suche nach theoretischen Rahmen als Grundlage zur Weiterentwicklung von Erziehungssowie Bildungszielen des Bürgers einer republikanischen Staatsform war. Deutsche Pädagogik in der ČSR wurde dagegen von einer sozialen sowie politischen „Desorientierung" des in den Böhmischen Ländern der Monarchie ursprünglich privilegierten Volkes gekennzeichnet, welches sich nach 28. Oktober 1918 im „fremden“ Staate der Tschechoslowaken befand. Die Flucht deutscher Pädagogik zum Geiste deutscher Kultur und zum deutschen Volkserbe (Volkstum, Volkheit) hatte auch historisch gerechtfertigt zu sein sollen. In diesem Sinne hatte die Geschichte der Pädagogik eine wichtige Rolle zu spielen und ist dabei auch erfolgreich gewesen.

\section{ANALysiertes LeHrbüCHer- KORPUS ZUR GESCHICHTE DER PädAgogIK FÜr PädAgogisChe INSTITUTE IN DER ČSR MIT TSCHECHISCH UND DEUTSCH ALS UNTERRICHTSSPRACHE}

Lehrbücher für Pädagogische Institute, welche im ganzen Bezugszeitraum als mittelschulische Anstalten galten, bedurften der Zustimmung des Ministeriums für Bildung und Nationalaufklärung. Voraussetzung dafür war eine unabhängige Evaluierung der Bücher durch die Fachgemeinschaft. Wurde ein Buch empfohlen, so folgte dessen Genehmigung als Lehrbuch für den jeweiligen Schultypus. Zum Zwecke der vorliegenden Studie habe ich alle Ausgaben des Amtsblattes des Ministeriums für Bildung und Nationalaufklärung untersucht (Jahre 1918-1939 und die danach folgenden 1939-1942), die vollständig in der National Bibliothek in Prag zur Verfügung stehen. Genehmigte Lehrbücher wurden am Ende jeder Ausgabe des jeweiligen Jahres veröffentlicht: Gesondert für Schulen mit Lehrsprache Tschechisch, Deutsch, Slowakisch, Ungarisch und/oder Polnisch und separat für Volks- und Bürgerschulen, Gesamtbildungsmittelschulen, Fachmittelschulen und Pädagogische Institute. Aus den vorliegenden Verzeichnissen geht klar hervor, dass es Pädagogischen Instituten erlaubt wurde, für bestimmte Unterrichtsfächer auch die für Mittelschulen genehmigten Lehrbücher einzusetzen. Lehrbücher für fachlichen Unterricht, zu dem auch Ge- 
genstände wie Methodik, Pädagogik oder Psychologie zählten, wurden ausschließlich für Pädagogische Institute genehmigt. Trotz der Untersuchung von insgesamt 22 Jahrgängen des ministerialen Amtsblattes, ist das Ergebnis in Bezug auf die Zahl der für Pädagogische Institute genehmigten Lehrbücher der Geschichte der Pädagogik überraschend. Für Pädagogische Institute mit Tschechisch als Unterrichtsprache wurde das Lehrbuch „Kurze Geschichte der Pädagogik" (Erstausgabe 1912) des Professors der Karls-Universität in Prag Otakar Kádner ${ }^{8}$ - genehmigt. Dasselbe Lehrbuch wurde im Zeitraum 1918-1939 insgesamt viermal herausgegeben (1920, 1922, 1926, 1931). ' Zwischen den Ausgaben 1918-1939 kann man keine bedeutsamen Unterschiede finden. Otakar Kádner war Pädagogik-Professor und jener, der im

${ }_{8}^{8}$ Otakar Kádner (1870-1936). Otakar Kádner zählt zu den bedeutendsten Vertretern zwischenkriegszeitlicher Pädagogik. 1906 habilitierte er in Prag mit seiner Schrift Beiträge zur experimentellen Pädagogik [Př́spèvky k pedagogice experimentální]. Seit 1907 war er Dozent im Pädagogischen Seminar der Karl-Ferdinands-Universität in Prag, welches er nach 1918 führte. Seit 1919 war O. Kádner als ordentlicher Professor der Philosophischen Fakultät der Karls-Universität tätig. Seine Forschung und Werk weisen starke positivistische Prägung auf. Der Fokus seiner fachlichen Aufmerksamkeit war auf experimentelle Pädagogik ausgerichtet, vor allem aber auf Allgemeine Pädagogik, Geschichte der Pädagogik und des Schulwesens. Sein Hauptwerk, welches für die Auffassung zwischenkriegszeitlicher Pädagogik bestimmend war, sind seine Grundlagen allgemeiner Pädagogik [Základy obecné pedagogiky] (I-III, 1925-1926), aber auch die dreibändige Geschichte der Pädagogik [Dějiny pedagogiky] (1909-1923). Die Rolle Kádners war nicht nur für die pädagogische Theorie und Forschung mitbestimmend. Zugleich war er aktiver Repräsentant einer ganzen Reihe von Vereinigungen und Organisationen, in denen sich eine aktive tschechoslowakische Lehrerschaft versammelte. So setzte sich O. Kádner erheblich für die Förderung von Ideen über hochschulische Lehrerbildung in der Tschechoslowakei der Zwischenkriegszeit ein, auch wenn dieses Ziel in der ČSR nicht zur Erfüllung kam. Darüber hinaus war O. Kádner im Bereich zwischenkriegszeitlicher Reform des Schulwesens und vor allem des Konzeptes einer einheitlichen, innen differenzierten Schule stark aktiv.

${ }^{9}$ Zusätzlich zu Kádners Lehrbuch wurden auch ältere Lehrbücher an der Wende zum 20. Jahrhundert genehmigt. Diese Bücher enthielten im Rahmen der Pädagogik auch einen historisch-pädagogischen Teil; z. B. das Lehrbuch von Alois Schlesinger - Kurze Erziehungslehre [Stručné vychovatelství] (1891, 1899, 1908), oder von František Švadlenka (1878-1931) - Allgemeine Pädagogik für Pädagogische Institute [Obecná paedagogika pro učitelské ústavy] (1913, 1920, 1921, 1928, 1929) und Allgemeine Didaktik für Pädagogische Institute [Obecná didaktika pro učitelské ústavy] (1912, 1921, 1924, 1929, auf Slowakisch 1924). Des Weiteren wurden Lehrbücher von G. A. Lindner verwendet, die - von Karel Domin überarbeitet, mehrfach herausgegeben wurden - Allgemeines Erziehungswesen: Lehrbuch für Institute zur Bildung von Lehrern und Lehrerinnen [Obecné vychovatelstvi: učebnice pro ústavy ku vzdèláni učiteli a učitelek] (1891, 1896, 1902, 1905, 1907,1909, 1911, 1914).

Historische Zusammenhänge von Bildung und Erziehung wurden auch durch Psychologie-Lehrbücher erörtert. Es handelt sich um Bücher von František Krejčí (1858-1934) - Psychologie (1897, 1919, 1921). Zur Ausbildung von Hauswirtschaftskunde- und Frauen-Handarbeiten-Lehrerinnen überwiegend auf Pädagogischen Instituten für Frauen oder in Frauen-Abteilungen von Pädagogischen Instituten wurde ein Lehrtext von Jan Adámek akkreditiert - Überblick über die Geschichte der Erziehung [Přehled dějin vychovatelství] (1921, 1923). Dieser Text ergänzte frühere Texte zur Bildung von Lehrerinnen von Josef Šauer (1845-1917) - Kurzes Erziehungs- und Lehrerwesen [Stručné vychovatelství a vyučovatelství] (1902, 1904) und Emanuel Hrys (1839-1906) - Kurzes Erziehungs- und Lehrerwesen zum Nutzen von Kandidatinnen des Lehrerberufs [Stručné vychovatelství a vyučovatelství k potřebě kandidátek učitelských] $(1897,1899)$. 
positivistischen Sinne in der tschechischen pädagogischen Debatte das Gebiet der Geschichte des Schulwesens und Bildung „beherrschte“. Diese Disziplin hat er vor allem in seinem dreibändigen Werk „Geschichte der Pädagogik“ (1909-1923) und teilweise auch in vier Bänden „Die Evolution und das heutige Bildungssystem“ (1929-1938) kodifiziert. Kádners positivistisch orientierte "Geschichte der Pädagogik" drängte die Bedeutung historisch-pädagogischer Erwägungen dessen Lehrers und Vorgängers auf der tschechischen Prager-Universität - František Drtina $^{10}$ - in den Hintergrund. Obwohl die historisch-pädagogischen Werke Professors F. Drtina hauptsächlich als Grundlage für das Universitätsstudium zukünftiger mittelschulischer Professoren dienten und für den Unterricht auf Pädagogischen Instituten nicht genehmigt waren, darf man Drtinas Einfluss auf das Geschichtsbild der Bildung und Erziehung auf Pädagogischen Instituten nicht unterschätzen. Lehrer an Pädagogischen Instituten als Absolventen der Prager-Universität verfügten über einen tiefen Einblick in Drtinas Werke und seine Auswirkung auf die nachwachsende MittelschullehrerGeneration. Ebenso beibehalten wurde der ausgeprägte aufklärerische Einfluss F. Drtinas auf die tschechische Volks- und Bürgerschullehrerschaft, und zwar sowohl in der Zeit vor dem Ersten Weltkrieg als auch nach der Gründung eines unabhängigen tschechoslowakischen Staates. O. Káder umfasst in seiner "Geschichte der Pädagogik" die Evolution pädagogischen Denkens seit der Antike bis zum Ende des Ersten Weltkriegs. Die insgesamt 5 Bände erstrecken sich über 1880 Textseiten, wobei ein bedeutender Teil des Werkes erst nach 1918 herausgegeben wurde. Die „Geschichte der Pädagogik“ des Prager Professors O. Kádner drängte das Werk des Professors der Pädagogik auf der MasarykUniversität in Brünn, Otokar Chlup ${ }^{11}$ „Ent-

\footnotetext{
${ }^{10}$ František Drtina (1861-1925) unterzog die historische Sichtweise der Erziehungs- und Bildungsfragen seiner Untersuchung im Werk Ideale der Erziehung [Ideály výchovy] $(1900,1925,1930)$ oder Gedankliche Entwicklung europäischer Menschheit [Myšlenkový vývoj evropského lidstva] (1902), als auch in seiner Entwicklung pädagogischer Theorien im 19. Jahrhundert [Vývoj theorii paedagogických v 19. století] (1903) oder Geschichte der Pädagogik unter besonderer Berücksichtigung allgemeiner kultureller und philosophischer Entwicklung [Dějiny paedagogiky se zvláštním zřetelem k povšechnému kulturnímu a filosofickému vývoji] (1905) oder auch in Entwicklung des Bildungswesen im 19. Jahrhundert [Vývoj školství v 19. stoleti] (1907).

${ }^{11}$ Otokar Chlup (1875-1965) promovierte an der Prager Philosophischen Fakultät als Student von Protagonisten tschechischer positivistischer Pädagogik und Psychologie (O. Kádner, F. Krejčí, F. Čáda). In der Zwischenkriegszeit war er als Pädagogik-Professor an der Masaryk-Universität in Brünn tätig. Seine anfänglichen Werke sind positivistisch orientiert. Er studierte moderne pädagogische Strömungen in England, in der Schweiz, in Frankreich und in Deutschland. Aus seinen Auslandsreisen hat er u. a. eines mitgebracht: Sein Interesse für experimentelle Pädagogik. Am pädagogischen Seminar in Brünn gründete er eine experimentelle Schule und war Repräsentant bei New Education Fellowship in der zwischenkriegszeitlichen Tschechoslowakei. Er widmete sich intensiv dem Studium amerikanischer Pädagogik. Im Streit um die Auffassung von Pädagogik, welcher in den 20. Jahren in der tschechischen pädagogischen Debatte eine Kulmination erlebte, stellte er sich dem Dozenten V. Pŕ́hoda und dessen markanter Zuneigung zum amerikanischen Pragmatismus und Behaviorismus entgegen. Er warnte vor dessen Einfluss auf die tschechische Pädagogik, deren tiefere Verankerung er in der europäischen Tradition sah. O. Chlup war stark linksorientiert und wurde zum ersten Dekan der Pädagogischen Fakultät der Karls-Universität in Prag nach 1945.
} 
wicklung pädagogischer Ideen im Neuen Alter" (1925) in den Hintergrund, welcher auf 200 Seiten besondere Aufmerksamkeit vor allem der Evolution neuzeitlichen pädagogischen Denkens widmet. Die Hälfte seines Textes analysiert hauptsächlich den positivistischen Einfluss auf die Pädagogik A. Comtes und H. Spencers. O. Chlup hat in seine Analyse in einem wesentlichen Ausmaß auch Werke tschechischer positivistisch- und experimentell-orientierten Pädagogen und Psychologen aus der Wendezeit vom 19. zum 20.
Jahrhundert miteinbezogen. Während Kádners "Geschichte der Pädagogik“ praktisch in der ganzen Zwischenkriegszeit als Grundlage für seine Vorträge auf der Prager-Universität dienten, ${ }^{12}$ wurde Kádners Lehrbuch „Kurze Geschichte der Pädagogik “ zur Norm in Pädagogischen Instituten.

Für deutschsprachige Pädagogische Institute gab es zwei genehmigte Lehrbücher. Autor des ersten Buchs „Geschichte der Erziehung und des Unterrichtes“, Theodor Tupetz, ${ }^{13}$ war bereits zur Zeiten 12 Nach O. Kádners Tod und bevor die tschechischen Hochschulen von deutschen NS-Besatzungsorganen im No-
vember 1939 geschlossen wurden, wurde Josef Hendrich (1888-1950) zum ordentlichen Professor der Pädagogik er-
nannt. J. Hendrich war bedeutender Comeniologe und wies eine reserviertere Haltung zur positivistisch konzipierten
Geschichte der Pädagogik auf. Er selbst versuchte, mit seinem Werk den Unterschied zwischen der Auffassung der Pä-
dagogik als empirische und exakte Wissenschaft auf der einen Seite, und als philosophische- oder Geisteswissenschaft
auf der anderen Seite, zu überbrücken. (Dies zeigt sich in Hendrichs Studien Einleitung in allgemeine Pädagogik [Üvod
do obecné pedagogiky], 1935 und Philosophische Strömungen in zeitgenössischer Pädagogik [Filosofické proudy v současné
pedagogice], 1926.) Auch wenn Hendrichs Universitätsvorträge zur Geschichte der Pädagogik nie in gedruckter Form
veröffentlicht wurden, geht aus der im Archiv durchgeführten Untersuchung dieser Unterlagen hervor, dass auch
Hendrich die Geschichte der Pädagogik als „unterstützende“ Disziplin zur Allgemeinen Pädagogik oder zur Theorie
der Erziehung versteht und dass er an Beispielen historischer „Figuren“ von Pädagogen Schlüsselfragen europäischer
Erziehungs- und Bildungstheorie sowie Praxis demonstriert.

${ }^{13}$ Theodor Tupetz (1852-1938) wurde in Fugau bei Schluckenau - in der nördlichst gelegener und heute verschwundener Gemeinde der Tschechischen Republik geboren. T. Tupetz studierte Geschichte (bei Anton Gindely), Geographie und Deutsche Philologie an der Prager-Universität. Nach seinen Studien wirkte er als Lehrer am Pädagogischen Institut in Prag, dann ab 1888 als Direktor des Pädagogischen Institutes in Trautenau. 1878 verteidigte er seine Doktorarbeit Der Türkenfeldzug von 1739 und der Friede zu Belgrad, welche auch veröffentlicht wurde. 1884 habilitierte er an der deutschen Prager Karl-Ferdinands-Universität mit der Arbeit Der Streit um die geistlichen Güter und das Restitutionsedict (1629). Im Jahre 1891 wurde er zum Landesschulinspektor für Volksschulen und Lehrerbildungsanstalten mit deutscher Unterrichtssprache und zum Landesschulrat ernannt. Er war Direktor der deutschen Sektion des Landesschulrats in Böhmen. T. Tupetz war Autor mehrerer Lehrbücher für Bürgerschulen, Gymnasien und Pädagogische Institute. Seine Geschichte- und Literaturlehrbücher erlebten zur Zeiten der Monarchie und später auch in deutschen Schulen der Tschechoslowakei eine große Verbreitung und Nutzung. Auch Tupetzs wissenschaftliche Arbeit in deutschen Gesellschaften Böhmischer Länder der Monarchie und in der Tschechoslowakei war von großer Bedeutung. Es handelte sich um seine Mitgliedschaft im Verein für die Geschichte der Deutschen in Böhmen und im Deutschen Kulturverband. Darüber hinaus gehörte er zu den Gründungsmitgliedern der Gesellschaft zur Förderung deutscher Wissenschaft, Kunst und Literatur in Böhmen, der späteren Deutschen Gesellschaft der Wissenschaft und Künste für die Tschechoslowakische Republik. Seit 1914 bekleidete er die Position des Vizepräsidenten der Deutschen Landeskommission für Kinderschutz und Jugendfürsorge, an deren Gründung er selbst Anteil hatte. 1921 wurde T. Tupetz pensioniert und starb 1938 in Prag. Der persönliche Nachlass T. Tupetzs befindet sich im Archiv des Denkmals des Nationalen Schrifttums 
der Habsburgermonarchie eine bekannte pädagogische Persönlichkeit. Er unterrichtete auf deutschen Pädagogischen Instituten. Tupetzs Publikation wurde 1891 erstmals veröffentlicht, eine nächste Ausgabe folgte nach $1918^{14}$. Autoren der zweiter Publikation, Lehrbuch der Pädagogik - III. Teil: Geschichte der Pädagogik (Erstausgabe 1922, dann 1929, 1933), waren der Universitätsprofessor an der
Deutschen Universität in Prag Wendelin Toischer ${ }^{15}$ und Anton Herget, ${ }^{16}$ Lehrer und später auch Direktor des Pädagogischen Instituts in Komotau (Chomutov), Autor einer umfassenden Studie zum Thema pädagogische Strömungen an der Wende vom 19. zum 20. Jahrhundert und eine bedeutende Persönlichkeit in der pädagogischen Debatte auf dem Gebiet der Kunsterziehung.

(Památník národního písemnictví) in Prag. Diese Daten habe ich aus dem Österreichischen Biographischen Lexikon bezogen (http://www.biographien.ac.at/oebl/oebl_T/Tupetz_Theodor_1852_1938.xml) sowie vom Petrbok, Václav, und Šolcová, Liběna (2005). Theodor Tupetz (1852-1938): Verzeichnis persönlichen Fonds [soupis osobniho fondu]. Prag: Památník národního písemnictví. Wichtig ist bei T. Tupetz seine Herausgeberschaft in der Reihe „Schulausgaben pädagogischer Klassiker“. In diesem Rahmen erschienen die folgenden Werke mit einem Vorwort von T. Tupetz: Lindner, Gustav Adolf. Allgemeine Erziehungslehre. Wien: A. Pichler's Witwe \& Sohn, 1894 und 1906. Milde, Vincenc Eduard. Allgemeine Erziehungskunde. Prag, Wien: F. Tempsky; Leipzig: G. Freytag, 1896. Salzmann, Christian Gotthilf. Krebsbüchlein. Prag, Wien: F. Tempsky; Leipzig: G. Freytag, 1896. Felbiger, Johann Ignaz. Methodenbuch. Prag, Wien: F. Tempsky; Leipzig: G. Freytag, 1896.

Comenius, Johann Amos. Orbis Pictus. Prag: Tempsky, 1896.

Pestalozzi, Johann Heinrich. Wie Gertrud ihre Kinder lehrt. Prag, Wien: F. Tempsky; Leipzig: G. Freytag, 1896. Rousseau, Jean Jacques. Emil oder Über Erziehung. Prag, Wien: F. Tempsky; Leipzig: G. Freytag, 1899.

Salzmann, Christian Gotthilf. Ameisenbüchlein. Prag, Wien: F. Tempsky; Leipzig: G. Freytag, 1900 und 1920.

${ }^{14}$ Eine historische Sichtweise auf die Erziehung war teilweise auch in weiteren Lehrbüchern zu finden, die für den Pädagogik-Unterricht an deutschen Pädagogischen Instituten in Böhmischen Ländern der Monarchie und später auch in der Tschechoslowakei akkreditiert waren. Es handelte sich um die wiederholte Ausgabe von G. A. Lindners Lehrbüchern, überarbeitet von Theodor Tupetz - Allgemeine Erziehungslehre (1894, 1906).

${ }^{15}$ Wendelin Toischer (1855-1922) war Gymnasialprofessor in Prag. Später habilitierte er bei O. Willmann in Prag und zwischen 1907-1922 bekleidete er die Funktion des ordentlichen Professors der Pädagogik an der Prager Karl-Ferdinands- und später Deutscher Universität in Prag. Als Nachfolger O. Willmanns würdigte er dessen Beitrag zur Pädagogik in hohem Maße. Er selbst jedoch widmete sich in seinen Vorträgen (bereits in den Jahren 1910-1914) vielmehr der Pädagogischen Psychologie, Neuen Strömungen in der Pädagogik, der Experimentellen Pädagogik, Themen individueller Pädagogik und des Talents.

${ }^{16}$ Anton Herget (1875-1944, Gabhorn bei Tepl-Prag) war Direktor des Pädagogischen Instituts in Komotau und Professor der Deutschen Musikakademie in Prag, Herausgeber des bedeutenden pädagogischen Periodikums Schaffende Arbeit, welches 1912 zum ersten Male erschien. Im Fokus der Zeitschrift lagen nicht nur Fragen der Kunsterziehung; aus einer breiteren Perspektive thematisierte sie auch Möglichkeiten der Schulreform und reflektierte ausgesuchte Erfahrungen aus experimenteller pädagogischer Arbeit.

Anton Herget gestaltete für Schüler deutscher Pädagogischer Institute eine dreiteilige Reihe von Pädagogik-Lehrbüchern. Der erste Teil war ein Psychologie-Lehrbuch, der zweite befasste sich mit Logik und der dritte, geschaffen in Co-Autorenschaft mit W. Toischer, wurde der Geschichte der Pädagogik gewidmet. Alle drei Teile stellten Lehrbücher vor, die für Leser und Schüler Pädagogischer Institute eine wesentliche Herausforderung in Bezug auf das Pensum des angebotenen Wissens und auf die Breite vorgestellter psychologischer und pädagogischer Themen dar. Der dritte Teil des mittelschulischen Lehrbuches wird im folgenden Teil dieses Artikels eingehender analysiert. 
3. UNTERSUCHUNG DER IN DEN

TSCHECHISCH- UND DEUTSCH-

SPRACHIGEN PÄDAGOGISCHEN

INSTITUTEN VERWENDETEN

LEHRBÜCHER ZUR GESCHICHTE

DER PÄDAGOGIK

Während die Erstausgabe von Tupetzs Geschichte der Erziehung und Unterricht bereits 1891 datiert, erschien das Lehrbuch Hergerts und Toischers erst im Jahre 1922. Sprich, es liegen mehr als 30 Jahre zwischen deren Erstpublikation, welche gerade in der Entwicklung pädagogischer Wissenschaft und des Unterrichtsfaches Pädagogik an Pädagogischen Instituten zu großen Veränderungen führten. Ein spezialisiertes Lehrbuch zur Geschichte der Pädagogik stand tschechischen Studenten erst nach 1912 zur Verfügung. Es war das Werk von O. Kádner. (Bis Dato nutzten Studenten historische Exkurse in älteren Pädagogik-Lehrbüchern, vor allem im Buch von F. Švadlenka, oder in G. A. Lindners überarbeiteten Lehrbüchern siehe Anmerkung 9.).

Das älteste zu analysierende Lehrbuch stammt aus dem Jahre 1891. Dessen Verfasser, T. Tupetz, war bereits ein habilitierter Dozent, Autor einer Reihe von Schulbüchern, ein erfahrener Lehrer und Direktor von Pädagogischen Instituten, als auch bedeutender Vertreter des deutschen schulpolitischen Lebens und relevanter Repräsentant des deutschen wissenschaftlichen Lebens in Böhmischen Ländern der Monarchie. T. Tupetz selbst konnte die "deutsche historische Schule“ in sich nicht leugnen: Seine "deutsche“ Schilderung der Geschichte Böhmischer Länder der Monarchie, wie von ihm wiederholter Weise in seinen Geschichtsbüchern präsentiert, wurde mit Erfolg „ausgesät" und verbreitet unter der deutschen Schülerschaft in deutschen Bürger- und Mittelschulen, vor allem zu Zeiten der Monarchie und teils auch in der Tschechoslowakischen Republik, wenn auch das republikanische Schulwesen auf dem Gebiet der deutschen Geschichtsbücher in sich eine große Konkurrenz darstellte. ${ }^{17}$ Liest man Tupetzs Geschichte der Erziehung und des Unterrichtes, so wird man auch auf dem Gebiet der Geschichte der Pädagogik mit einer ähnlichen „nationalen" Anschauung konfrontiert. Die Geschichte der Pädagogik wird da als eine außerordentlich bedeutende Disziplin und ein typisch deutsches pädagogisches Fach vorgestellt. Dem entspricht auch die Auswahl jener in den Saal des Ruhmes der Geschichte der Pädagogik aufgenommen Repräsentanten-Klassiker. Einen beträchtlichen Teil davon stellen Vertreter des deutschen Pietismus und vor allem des

\footnotetext{
${ }^{17}$ In der zwischenkriegszeitlichen, demokratischen und republikanischen Tschechoslowakei stand die Auffassung der Geschichte als Unterrichtsfaches im Mittelpunkt der pädagogisch-technischen Debatte. Es ist angesichts des Interesses eines republikanischen, multinationalen Staates darum gegangen, eine wissenschaftlich noch kritischere Grundlage zu schaffen. Daher genehmigte das zwischenkriegszeitliche Bildungsministerium im Gegensatz zu den Lehrbüchern von Tupetz, eine große konkurrierende Zahl an Lehrbuchtiteln, die die Geschichte Böhmischer Länder anhand neuerer Ergebnisse historischer Wissenschaft präsentierten.
} 
Philanthropismus dar, wobei die gesamte Evolution des preußischen Schulwesens als Deutsch betrachtet wird. Auf eine ähnliche Weise wird die Geschichte der Pädagogik auch vom Team A. Hergert und W. Toischer erzählt. Auch in deren Lehrbuch wird die Evolution des österreichischen Schulwesens als ein exklusives Bild preußischer Schultradition und des deutschen Philanthropismus dargestellt. Auch wenn es nicht bestritten werden kann, dass sich österreichisches Bildungswesen zum Ende des 18. Jahrhunderts erheblich aufdie pädagogische Tradition des Philanthropismus stützte, muss ich darauf hinweisen, dass weder T. Tupetz, noch A. Hergert oder W. Toischer Prozesse des „Transfers“ philanthropischer Ideen aus dem deutschen in den österreichischen Raum thematisieren (sprich in die tschechische als auch in die deutsche pädagogische Debatte in Böhmischen Ländern der Monarchie). Es wird von den Autoren nicht hinterfragt, wie der philanthropische Einfluss von den Vertretern der Pädagogik in Böhmischen Ländern aufgenommen und inwieweit aktiv unterstützt wurde. Offensichtlich hatte der Leser den Eindruck zu bekommen, dass das Bildungswesen in Böhmischen Ländern der Monarchie (das tschechische als auch das deutsche) eine Art Abguss des preußischen war und vor allem, dass die Entwicklung der pädagogischen Debatte in Böhmischen Ländern der Monarchie durch eine einseitige Übernahme weiter verlief. Man hat den Leser nicht zum Bewusstsein hingeführt, dass pädagogische Diskussion aus verschiedenen Prozessen aufgebaut wird, die Kontinuität aber auch
Diskontinuität enthalten. Sowohl T. Tupetz, als auch A. Herget und W. Toischer arbeiten mit der Vorstellung einer geradlinigen Entwicklung der Wissenschaft und nicht mit einer „revolutionären“ Gestaltung wissenschaftlicher Paradigmen. Eine ähnliche Anschauung findet man auch in Kádners tschechischem Lehrbuch. Auch O. Kádner, als klassischer und markanter Vertreter des tschechischen Positivismus, arbeitet mit einem Fortschritts- und entwicklungsorientierten Konzept der Evolution des pädagogischen Denkens.

Sowohl tschechisch- als auch deutschsprachige Lehrbücher versicherten im Geiste des Positivismus Studenten Pädagogischer Institute, dass die Evolution der Pädagogik als Wissenschaft auf einfache und weniger ausgearbeitete Theorien des Altertums oder der Antike zurückgeht, über das Mittelalter und Neuzeit bis zu ihrem Höhepunkt in der Aufklärung, und zur modernen Verankerung des pädagogischen Denkens. Nichtsdestotrotz machen sich bestimmte Unterschiede zwischen der Überlieferung der Entwicklung des pädagogischen Denkens in tschechisch- und deutschsprachigen Lehrbüchern bemerkbar. Einerseits sind sie auf die „nationale oder national-emanzipatorische Aufgabe“ der Pädagogik im Bezugszeitraum zurückzuführen, anderseits aber auch auf die unterschiedliche Entwicklung der Pädagogik als Wissenschaft in beiden nationalen Debatten.

Die bereits erwähnte „nationale“ Lesart der Entwicklung pädagogischen Denkens bedeutete, dass sich z. B. nicht nur das Werk von J. A. Komenský in den 
tschechischen Saal des Ruhmes durchkämpfen konnte, welches eine Aufmerksamkeit auch in deutschen Lehrbücher verdiente, sondern auch und in einem höheren $\mathrm{Maß}$ weitere humanistische $\mathrm{Au}$ toren und vor allem moderne tschechische Pädagogen. Otakar Kádner machte mit Stolz die angehende Lehrer-Generation mit Ergebnissen der damaligen pädagogischen Forschung tschechischer SpitzenPädagogen (F. Krejčí, F. Čáda) bekannt, sowie mit breiteren Universitätsdebatten an der tschechischen Prager-Universität (das Werk von T. G. Masaryk, O. Hostinský, F. Drtina), oder mit der Bedeutung aktiver Lehrer (J. Klika, E. Makovička, A. Janů, J. Mrazík, J. Úlehla, J. Černý), die eine wesentliche Rolle nicht nur in der Vereinigung der Lehrerschaft gespielt haben, sondern auch in der Überlieferung von Ergebnissen pädagogischer Forschung im In- und Ausland an die tschechische Lehrerschaft (egal ob durch Veröffentlichung popularisierender Publikationen, relevanter pädagogischer Zeitschriften oder mit präzisen Übersetzungen ausländischer Pädagogen). Es steht auch außer Zweifel, dass Kádners Publikation die russische, polnische, südslawische, italienische, aber auch die französische, englische oder amerikanische Pädagogik stärker in den Blickpunkt gerückt hat. Des Weiteren kommt in Kádners Publikation ein Merkmal des Autors klar zum Ausdruck: In seinen weiteren Publikationen achtete Kádner auf einen komparativen Zugang und analysierte Phänomene und Probleme aus verschiedensten Betrachtungswinkeln. Sprich, aus der Perspektive verschiedener Schulen und Theorien und daher auch aus verschiedenen „nationalen“ Blickwinkeln. Ebenso wurde österreichische Pädagogik und österreichische Schule in Kádners Betrachtungsweise nicht als deutsche Schule und schon gar nicht als eine Schule nach preußischem Muster präsentiert. Österreichische Schule wurde als eigenständige, aus eigener pädagogischer Entwicklung und pädagogischer Debatte in einer multinationalen Lage der Monarchie resultierende Institution geschildert, was später auch auf die ČSR zutraf. Selbstverständlich bestreitet O. Kádner keineswegs den bedeutenden Einfluss deutscher Pädagogik auf das österreichische Bildungswesen. Dennoch vergisst er nicht, eine Analyse der sich auf dem Gebiet der Monarchie entwickelnden pädagogischen Diskussion anzubieten. Auf diese Weise bewirkt er nicht nur eine Abschwächung der „Überschreibung“ der Geschichte der Pädagogik in Böhmischen Ländern der Monarchie durch deutsche Tradition, sondern er berücksichtigt noch stärker eine realistischere und diversifiziertere Bildung pädagogischer Tradition in der ČSR.

Die Schwachstelle des Lehrbuches von T. Tupetz sowie dessen neuerer Ausgaben lag in der Tatsache, dass es in der Publikation gar keine Hinweise auf die Entwicklung pädagogischen Denkens ab der zweiten Hälfte des 19. Jahrhunderts zu finden waren. Das Buch vermittelte zwar Studenten Auskunft über die Schule von V. E. Milde und natürlich auch über den ,imposanten und entscheidenden Einfluss“" J. H. Pestalozzis; dennoch wurden jene 
Ereignisse, die auf dem Gebiet der Erziehung und des Bildungswesens nach 1848 eintraten, im Lehrbuch negiert und praktisch verdrängt. Die Gesamtschilderung der Pädagogik bei T. Tupetz geht also von einer rein deutschen Tradition und derer unerschütterlichen Position aus. Was den Umfang vom Tupetzs Buch betrifft, sind zwei Drittel aller Seiten der deutschen Reformation und deren Einfluss auf das Schulwesen sowie deutschem Pietismus, Philanthropismus und dem Werk von Pestallozzi und Diesterweg gewidmet. Der Saal des pädagogischen Ruhmes wurde also völlig mit der deutschen pädagogischen Tradition besetzt, ohne dass andere eine Einladung bekommen hätten. Das Lehrbuch der Autoren A. Herget und W. Toischer wirkte schon aus der Sicht chronologischer Entwicklung moderner und insgesamt ausgewogener. Auch wenn die Beherrschung deutscher "pädagogischer Kultur" in diesem Werk auf der Hand liegt, als auch ihre Dankbarkeit gegenüber der deutschen klassischen Philosophie, weisen dessen Autoren die Leser auf weitere Persönlichkeiten und Tatsachen der pädagogischen Debatte sowie des Schullebens in Böhmischen Ländern der Monarchie hin. Auf der anderen Seite bleiben auch pädagogische Debatten in anderen Ländern oder Regionen Europas in diesem Buch unerwähnt und die Dominanz deutscher Pädagogik ist auch hier außerordentlich stark. Tschechische Pädagogik, die nach 1882 einen herausragenden Entwicklungsgrad erreichte, wurde in diesem Werk gar nicht reflektiert oder thematisiert. Auf diese Weise „bestritten“ Autoren des deutschen Lehrbuchs den zumindest zweisprachigen Charakter Böhmischer Länder der Monarchie und drängten die tschechische Wissenschaft aus ihrer pädagogischen Reflexion hinaus. Natürlich lag der Grund hierfür in der Unmöglichkeit, tschechische Fachliteratur zu lesen, da sie seit dem letzten Drittel des 19. Jahrhunderts auf Tschechisch herausgegeben wurde, d. h. in einer Sprache, die die deutsche wissenschaftliche Elite des Bezugsraumes bis auf Ausnahmen (Bohemisten) nicht verstanden hat. Nur wenn deutsche Übersetzungen oder Auszüge und Artikel tschechischer Autoren auf Deutsch veröffentlicht wurden, hatte die deutsche pädagogisch-wissenschaftliche Elite in Prag und an deutschen Pädagogischen Instituten die Möglichkeit, sich ein ausreichend realistisches Bild über die Ausrichtung der böhmischen pädagogischen Debatte zu machen. Tschechische Pädagogen dagegen lasen deutsche sowie weltrelevante pädagogische Werke problemlos, was ihnen einen Vergleich einzelner pädagogischer Schulen ermöglichte. So konnte die angehende tschechische Lehrergeneration sowohl mit der deutschen, als auch mit der weiteren Entwicklung der pädagogischen Debatte in Europa und der Welt bekannt gemacht werden. Hiermit konnten sich tschechische Pädagogen von der spezifischen Grundlage deutscher Pädagogik in österreichischen Ländern distanzieren, die sich erheblich auf deutsche Philosophie stützte. Das hat sich u. a. in einer unterschiedlichen Konzeption der Pädagogik als Wissenschaft gespiegelt, die in der tschechischen und deutschen päda- 
gogischen Debatte zum Ausdruck kam, wie bereits im ersten Teil dieses Artikels kurz erwähnt. Am deutlichsten zeigt sich diese Tatsache in Toischers und Hergets Lehrbuch in der Beschreibung moderner pädagogischer Richtungen an der Wende zum 20. Jahrhundert. Die Liste reformpädagogischer Richtungen in diesem Lehrbuch schließt ein: Kunsterziehung (A. Lichtwark, E. Weber), Arbeitsschule (G. Kerschensteiner, H. Gaudig), staatsbürgerliche Erziehung (G. Kerschensteiner, F. W. Foerster, Konzept der Schulgemeinschaft), Sozialpädagogik (P. Natorp, O. Willmann), Individualpädagogik (L. Gurlitt, E. Key, B. Ottto), Moralpädagogik (F. W. Foerster, P. Barth, W. Börner) und experimentelle Pädagogik (E. Meumann, A. Lay, W. Stern). ${ }^{18}$ Ausländische Pädagogen weisen in der
Liste keine stärkere Präsenz auf und die Schulreform an der Wende zum 20. Jahrhundert wird bei A. Herget und W. Toischer wieder als deutsche Angelegenheit vorgestellt. Niemand außer deutsche Pädagogen waren hiernach dazu befugt, ein Schulreform „Rezept“ anzubieten.

O. Kádners Lehrbuch thematisiert "neue Richtungen“ nicht als ein gesondertes Kapitel. Es versteht sie als Ergebnis oder Äußerung des Nachdenkens über die Ausrichtung pädagogischer Wissenschaft, die sich vielmehr an Ergebnissen empirischer Forschung, an der Suche nach Antworten auf soziale und gesellschaftliche Bedürfnisse einer sich schnell modernisierenden Gesellschaft, einer demokratischen und republikanischen Gesellschaft und einer national-gestalteten Gesellschaft zu stützen hatte. ${ }^{19}$

\footnotetext{
${ }_{18}$ Anton Herget definiert den „Kanon“ reformpädagogischer Richtungen bereits in seinem Werk Die wichtigsten Strömungen im pädagogischen Leben der Gegenwart, welches 1915 in zwei Teilen erschien. In deren Rahmen bezieht Herget in die „neuen pädagogischen Strömungen“, die sich gegen die "alte Schule“ oder Buchschule stellen, jene pädagogischen Richtungen mit ein, mit denen er sich im dritten Teil seines Lehrbuches für Pädagogische Institute befasst. Es handelte sich um Lichtwarks Kunsterziehung, Kerschensteiners Arbeitsschule, Individualpädagogik bei L. Gurlitt und H. Lietz, P. Geheeb, G. Wyneken, um die Schule von B. Otto, die natürliche Erziehung von E. Haufe, um die Experimentalpädagogik, Moralpädagogik von F. W. Foerster und P. Natorp und um das Phänomen der deutschen Jugendbewegung.

Hergets Studie, wiederholter Weise publiziert, wurde zu einem breit rezipierten Werk in der Habsburgermonarchie, aber auch in der deutschen Pädagogik.

${ }^{19}$ Ergebnisse bisheriger Forschung über die Formulierung eines Reformprogrammes für das Schulwesen sowohl unter Lehrern von Grund- und Bürgerschulen, als auch unter der akademisch-pädagogischen Fachöffentlichkeit deuten darauf hin, dass Forderungen nach einer Reform zwischenkriegszeitlicher "tschechischer" Schule unter anderem durch zwei Faktoren legitimiert wurden: Durch Argumente historischer Entwicklung tschechischer Schule und Entwicklung pädagogischen Denkens, und durch das Bestreben nach Schaffung einer republikanischen Schule. Reformziele der tschechischen Schule hatten sich deutlicher an exakte Ergebnisse der Forschung über Kinder anzulehnen, während in der deutschen pädagogischen Tradition der Tschechoslowakei eine Argumentation überwog, die durch Kulturfragen und durch den sogenannten Geist der Zeit legitimiert wurden. Eine Untersuchung des Reformprogrammes des Schulwesens in der zwischenkriegszeitlichen tschechischen pädagogischen Debatte findet im Rahmen eines Projektes unter staatlichen Beihilfe der Agentur GAČR The analysis of pedagogical concepts of school reform in inter-war Zlin in the context of a model industrial company town Reg. Nr. 16-13933-S statt.
} 


\section{FAZIT}

Wie aus der Analyse tschechischer und deutscher Lehrbücher zur Geschichte der Pädagogik für Pädagogische Institute hervorgeht, stellte das Unterrichtsfach Geschichte der Pädagogik ausschließlich ein Gebiet der Geschichte des pädagogischen Denkens vor. Eine sozialhistorische Untersuchung zur Geschichte der Bildung und Erziehung war in den analysierten Lehrbüchern nicht vorhanden und stellte keinen Bestandteil des Curriculums mittelschulischer Lehrerbildungsinstitute dar.

Es lässt sich sagen, dass die Geschichte der Pädagogik als Disziplin und ihr Inhalt in den berücksichtigten Lehrbuch-Beispielen - und vor allem in den deutschen - fix verankert und durch ihren funktionellen Charakter gekennzeichnet war. Auf verschiedenen Ebenen legitimierte sie die Entwicklung des Bildungswesens sowie die Anschauung pädagogischer Debatte, wie diese gegen Ende des 19. Jahrhunderts in der Habsburgermonarchie aufgebaut wurde.

Das tschechische Lehrbuch Kádners präsentiert die Geschichte der Pädagogik wiederum mit größerer Offenheit, da Änderungen in der Auffassung der Pädagogik als Wissenschaft Ende des 19./Anfang des 20. Jahrhunderts gleichzeitig einen neuen Blick auf die historisch-pädagogische Disziplin öffneten. Kádner schildert Reformströmungen an der Wende zum 20. Jahrhundert nicht als ein "Chaos", sondern verknüpft mit der Eröffnung einer breiteren gesellschaftlichen und multidisziplinar-wissenschaftlichen (Psy- chologie, Soziologie, Philosophie, Anthropologie) Debatte über Aufgaben, Ziele, Mittel und Bedingungen der Erziehung und der Bildung. Auch wenn Kádner die Entwicklung des Faches aus der Sicht eines positivistischen Gesetzes betrachtete, wurde der Focus durch die vom Autor in seine Schilderung der Geschichte der Pädagogik ins Lehrbuch miteinbezogenen Ergebnisse experimenteller Pädagogik, Psychologie, pädagogischen Psychologie und weiterer angrenzenden Wissenschaften aus einem festgelegten Bereich pädagogischen Denkens auf die Geschichte der Pädagogik gerichtet. Trotzdem ist festzustellen, dass sowohl deutsche als auch tschechische Lehrbücher zur Geschichte der Pädagogik im Leser ein verhältnismäßig „stabilisierendes“ Gefühl vom studierten Fach hinterließen. Für den Leser war es offensichtlich, dass es sich um einen klar erforschten Zweig pädagogischer Wissenschaft handelt, der auf keine Weise in Frage gestellt, niedergerissen oder kritisch gesehen werden darf. Bei dem zu untersuchenden Beispiel kann der normative Charakter der Disziplin „Geschichte der Pädagogik" nicht übersehen werden, eher umgekehrt. Es geht eindeutig hervor, dass die Geschichte der Pädagogik ihre Verankerungsaufgabe für Pädagogik als Wissenschaft zu erfüllen hatte und das die „königliche“ Disziplin „Geschichte der Pädagogik" die junge Disziplin und deren Historie zu legitimieren hatte. Die „Mission“ der Geschichte der Pädagogik in ihrer gestalterischen Rolle einer modernen pädagogischen Wissenschaft war erheblich. Es waren Fakten gefragt, 
reichlich und repräsentativ, damit sie als Grundlage für die Disziplin der Geschichte der Pädagogik dienen konnten. Pädagogisches Denken und pädagogische Persönlichkeiten wurden auf normative Weise als festes Skelet der Geschichte der Pädagogik ,gestaltet" und konnten ihren unerschütterlichen Charakter bis zum Anfang des Zweiten Weltkriegs behalten. Die Disziplin der Geschichte der Pädagogik schrieb also eine „eigene Geschichte der Geschichte der Pädagogik“.

Außerdem wird es deutlich, dass die Geschichte der Pädagogik auch Merkmale ihrer nationalen Aufgabe aufwies. $\mathrm{Zu}$ Beginn des 20. Jahrhunderts und in der ČSR hat man Wissenschaft als einen aus den für die Entwicklung nationalen Lebens relevantesten Bereichen verstanden. In einer Atmosphäre des nationalen Wettbewerbs bis hin zu einem Nationalkampf, welcher typisch für den Bezugszeitraum in Böhmischen Ländern war, stellen wir fest, dass auch die Geschichte der Pädagogik von diesem Einfluss nicht verschont blieb. Als Beispiel dient J. A. Comenius, welcher sowohl in tschechischen als auch in deutschen Lehrbüchern inhaltlich vertreten war. Während im böhmischen Bereich seine Bedeutung für die tschechische Sprache und für die Gestaltung einer tschechischen „nationalen Identität“ hervorgehoben wurde und er als Beispiel tschechischen „Widerstandes“ gegen die habsburgische, sprich katholische, also deutsche Kultur präsentiert wurde, stellten deutsche Lehrbücher Comenius vor allem als Nachfolger deutscher Didaktiker dar. Als einen, aus dem deutschen Re- formdenken schöpfenden Repräsentanten und Gründer der modernen Schule der Neuzeit, so wie es später auch deutsche Philantropisten taten.

Angesichts des fehlenden Zeitrahmens sowie der fehlenden breiteren $\mathrm{Zu}$ sammenhänge der Auffassung des Bildungsniveaus sowohl in tschechischen als auch deutschen Lehrbüchern, war es weitaus einfacher, einzelne Persönlichkeiten der "Geschichte der Pädagogik“ für die jeweiligen Bildungsziele zu instrumentalisieren. Ich vermute jedoch, dass es für die angehenden Lehrer von keiner so prinzipiellen Bedeutung war, wie man die einzelnen Persönlichkeiten schilderte, da angesichts der Fülle von „Fakten“ und der positivistischen Schilderungsart pädagogischer Richtungen und Figuren, Lehrer und Studenten gewiss einiges von den dort beschriebenen Umständen entweder vergessen oder verdrängt haben. Weitaus schwerwiegender ist es, dass man ihnen nicht beigebracht hat, wie man die präsentierten Gegebenheiten in breiteren $\mathrm{Zu}$ sammenhängen zu sehen hat. Also haben sie sich auch keine Fragen darüber gestellt, welche pädagogische Überzeugung jeder der großen Pädagogen vertrat und weshalb. So kam es dazu, dass wissenschaftliche und nationale Stereotypen von jener bedeutenden sozialen Gruppe - sprich von Lehrern der Grund- und Bürgerschulen übernommen wurden, die angesichts der damaligen Struktur und Prozesse in der Gesellschaft der Zwischenkriegszeit keinen geringen Einfluss auf die Ausbildung der Verhaltensweisen junger Generation hatte. 
Zusammenfassend ist festzustellen, dass alle Funktionen, die man der Geschichte der Pädagogik als Disziplin zuschreiben kann, im Laufe der gesamten Zwischenkriegszeit und in beiden Sprachlagern Großteils ihre Anwendung fanden. Die tschechische historisch-pädagogische Reflexion ist 1939 nach der Schließung der tschechischen Hochschulen mit Gewalt verstummt und die deutsche Geschichte der Pädagogik musste in Übereinstimmung mit ideologischen Zielen des deutschen Nationalsozialismus im Protektorat Böhmen und Mähren in Einklang gebracht werden. Nach Ende des Zweiten Weltkriegs kam es in Mitteleuropa de facto zur Verdrängung der deutschen Geschichte der Pädagogik aus der Karte Europas. Es ging aber noch weiter: Das Pädagogische Gedächtnis und das getrennte Europa haben sie zur Vergessenheit verurteilt. Die tschechische Schreibweise der Geschichte der Pädagogik geriet bald unter das Diktat der marxistisch-leninistischen Ideologie und deren Erkenntnistheorie, welche für die Betrachtung der Erziehung für das kommunistische Regime in der Tschechoslowakei eine erhobene Bedeutung hatte. Auch nach 1989 kommt es zu keiner intensiveren „Neuschreibung“ der traditionellen oder „alten“ Schulen der Geschichte der Pädagogik und die tschechische pädagogische Historiographie findet nur langsam und mit Mühe Anknüpfungspunkte zur postmodernen Konzeption „neuer“ Geschichte der Pädagogik. Eine Entmythologisierung, Entmystifizierung und Dekonstruktion vieler der festgelegten und „mit Selbstverständlichkeit gültigen historisch-pädagogischen" Bauwerke stellte für das Forschungsinteresse in der tschechischen pädagogischen Debatte nur ein marginales Thema dar. Einige Arbeiten im Bereich Sozial- und Kulturgeschichte der Alltäglichkeit spielen in dieser Hinsicht eine positive Rolle. Es stellt sich jedoch die Frage, inwieweit solche Ergebnisse einen tatsächlichen Einfluss im Lager der mit der Erziehung der zukünftigen Lehrerschaft beauftragten Pädagogen bewirken, damit sich auch diese mit dem Ansatz eines „new cultural historian of education" konfrontiert sehen (Cohen, 1999; Depaepe, 2017). Folglich ist es von Bedeutung sich die Frage zu stellen, inwieweit die tschechische Pädagogik eine weitere Transformation „pädagogischer Ideen und Wahrheiten“ unterstützt und inwieweit sie in der Lage ist, bei den angehenden Lehrern eine offene historische Reflexion über pädagogische Ereignisse, Gedanken und Vorkommnisse in Sinne der critical hermeneutics (Depaepe, 2017) zu entwickeln, die sie bei ihrer täglichen Arbeit in der Schulklasse einsetzen könnten.

Ein Teil dises Beitrages entstand mit der Unterstützung der Grantová Agentura $\check{C} R$, der staatlichen Forschungsförderungsagentur der Tschechischen Republik, im Rahmen des Projektes Nr. 16-13933S The analysis of pedagogical concepts of school reform in inter-war Zlin in the context of a model industrial company town. 


\section{Quellen:}

Herget, A. (1920). Die wichtigsten Strömungen im pädagogischen Leben der Gegenwart. I. Teil (4., erweiterte Auflage). Prag: A. Haase.

Herget, A. (1921). Die wichtigsten Strömungen im pädagogischen Leben der Gegenwart. II. Teil (4., erweiterte Auflage). Leipzig: A. Haase.

Herget, A., \& Toischer, W. (1922). Lehrbuch der Pädagogik. 3. Teil, Geschichte der Pädagogik. Prag: A. Haase.

Kádner, O. (1922). Stručné dějiny pedagogiky (3. Ausg.). Prag: Česká grafická Unie.

Tupetz, T. (1891). Geschichte der Erziehung und des Unterrichtes: Lehrbuch für den IV. Jahrgang der österreichischen Lehrer- und Lehrerinnenbildungsanstalten. Prag: F. Tempsky.

\section{Literatur:}

Cohen, S. (1999). Challenging orthodoxies: towards a new cultural history of education. New York: Lang. Depaepe, M. (2001). A professionally relevant history of education for teachers: does it exist? Reply to Herbst's the State of the Art Article. Paedagogica Historica, 37, 631-640.

Depaepe, M. (2017). More than an antidote against amnesia... Some historiographical, theoretical, and methodological reflections on the History of Education. Pedagogika, 67(3), in press.

Kasper, T., \& Kasperová, D. (2013). Die berufliche und nationale Emanzipation der tschechischen Lehrerschaft im letzten Drittel des 19. Jhrs. Motive, Ziele, Erfahrungen und Reflexionen. History of Education and Children's Literature, 8(1), 181-198.

Kasper, T., \& Kasperová, D. (2015). National, state and civic education in the Czech Lands of Austro-Hungarian Empire in Czechoslovakia after 1918. History of Education and Children's Literature, 10(1), 251-278.

Kasper, T., \& Kasperová, D. (2017). Exactness and Czech pedagogy at the break of the 19th and 20th century. History of Education and Children's Literature, 12(2), in press.

Tenorth, H. E. (1996). Lob des Handwerks. Kritik der Theorie - Zur Lage der pädagogischen Historiographie in Deutschland. Paedagogica Historica, 32(2), 343-361.

Tröhler, D. (2014). The construction of society and conceptions of education. Comparative visions in Germany, France, and the United States around 1900. In T. S. Popkewitz (Ed.), The reason of schooling: Historicizing curriculum studies, pedagogy, and teacher education (S. 21-39). New York, NY: Routledge.

Tröhler, D. (2017). Tracking the educationalization of the world: Perspectives for an emancipated history of education. Pedagogika, 67(3), in press.

Doc. PhDr. Tomás Kasper, Ph.D.

Technical University of Liberec, Faculty of Sciences, Humanities and Education, Department of Pedagogy and Psychology;

e-mail: tomas.kasper@tul.cz 